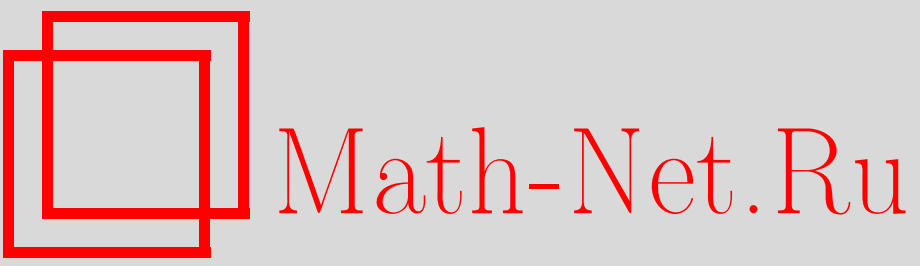

О. И. Мохов, Нелокальные гамильтоновы операторы гидродинамического типа с плоскими метриками и уравнения ассоциативности, УМH, 2004, том 59, выпуск 1, 187-188

DOI: https://doi.org/10.4213/rm712

Использование Общероссийского математического портала Math-Net.Ru подразумевает, что вы прочитали и согласны с пользовательским соглашением

http://www.mathnet.ru/rus/agreement

Параметры загрузки:

IP: 54.237 .206 .68

26 апреля 2023 г., 12:34:30 


\title{
НЕЛОКАЛЬНЫЕ ГАМИЛЬТОНОВЫ ОПЕРАТОРЫ ГИДРОДИНАМИЧЕСКОГО ТИПА С ПЛОСКИМИ МЕТРИКАМИ И УРАВНЕНИЯ АССОЦИАТИВНОСТИ
}

\author{
О.И. Мохов
}

Мы решаем задачу описания всех нелокалных гамилтоновых операторов гидродинамического типа с плоскими метриками. Доказано, что в ряде важных случаев такие гамилтоновы операторы описьваются уравнениями ассоциативности и тесно связаны с теорией фробениусовых многообразий. Напомним, что в [1] Е. В. Ферапонтов ввел и изучил общие нелокалшные гамильтоновы операторы гидродинамического типа, а именно, гамильтоновы операторы вида

$$
P^{i j}=g^{i j}(u(x)) \frac{d}{d x}+b_{k}^{i j}(u(x)) u_{x}^{k}+\sum_{n=1}^{L} \varepsilon^{n}\left(w_{n}\right)_{k}^{i}(u(x)) u_{x}^{k}\left(\frac{d}{d x}\right)^{-1} \circ\left(w_{n}\right)_{s}^{j}(u(x)) u_{x}^{s},
$$

где $\operatorname{det}\left(g^{i j}(u)\right) \not \equiv 0, \varepsilon^{n}= \pm 1,\left(u^{1}, \ldots, u^{N}\right)$ - локальные координаты, $u^{i}(x)$ - полевые функции. В [1] доказано, что оператор (1) является гамильтоновым тогда и только тогда, когда 1) $b_{k}^{i j}(u)=-g^{i s}(u) \Gamma_{s k}^{j}(u)$, где $\Gamma_{s k}^{j}(u)$ - риманова связность, порождаемая контравариантной метрикой $g^{i j}(u)$ (связность Леви-Чивита), 2) метрика $g^{i j}(u)$ и афффиноры $\left(w_{n}\right)_{j}^{i}(u), 1 \leqslant n \leqslant L$, удовлетворяют следующим соотношениям: $g_{i k}(u)\left(w_{n}\right)_{j}^{k}(u)=g_{j k}(u)\left(w_{n}\right)_{i}^{k}(u), \nabla_{k}\left(w_{n}\right)_{j}^{i}(u)=$ $\nabla_{j}\left(w_{n}\right)_{k}^{i}(u)$, где ковариантная производная определяется связностью Леви-Чивита метрики $g^{i j}(u), R_{k l}^{i j}(u)=\sum_{n=1}^{L} \varepsilon^{n}\left(\left(w_{n}\right)_{l}^{i}(u)\left(w_{n}\right)_{k}^{j}(u)-\left(w_{n}\right)_{l}^{j}(u)\left(w_{n}\right)_{k}^{i}(u)\right)$, где $R_{k l}^{i j}(u)$ - тензор римановой кривизны метрики $g^{i j}(u)$, и, кроме того, семейство афффиноров $w_{n}(u), 1 \leqslant n \leqslant L,-$ коммутативное: $\left[w_{n}, w_{m}\right]=0$.

Рассмотрим важный специальный случай, когда метрика $g^{i j}(u)$ - плоская. Напомним, что любая плоская метрика определяет однозначно локальный гамильтонов оператор гидродинамического типа (оператор вида (1) с нулевыми афффинорами) - гамильтонов оператор Дубровина-Новикова [2]. Но мы доказьваем, что имеется и богатый класс нелокальных гамильтоновых операторов гидродинамического типа с плоскими метриками, причем имеющих важные приложения в теории фробениусовых многообразий и интегрируемых иерархий. Вид гамильтонова оператора (1) инвариантен относительно локальных замен координат, при этом все коэффициенты оператора преобразуются как соответствующие дифференциально-геометрические объекты. Так как метрика плоская, то существуют локальные координаты, в которых метрика приводится к постоянному диагональному виду $\epsilon^{i} \delta^{i j}, \epsilon^{i}= \pm 1$. В этих локальных координатах все коэффициенты связности Леви-Чивита равны нулю и гамилттонов оператор имеет вид:

$$
\widetilde{P}^{i j}=\epsilon^{i} \delta^{i j} \frac{d}{d x}+\sum_{n=1}^{L} \varepsilon^{n}\left(\widetilde{w}_{n}\right)_{k}^{i}(u(x)) u_{x}^{k}\left(\frac{d}{d x}\right)^{-1} \circ\left(\widetilde{w}_{n}\right)_{s}^{j}(u(x)) u_{x}^{s} .
$$

Теорема 1. Oператор (2) является гамильтоновым тогда и только тогда, когда существуют функции $\psi_{n}(u), 1 \leqslant n \leqslant L$, такие, что

$$
\left(\widetilde{w}_{n}\right)_{j}^{i}(u)=\epsilon^{i} \frac{\partial^{2} \psi_{n}}{\partial u^{i} \partial u^{j}}
$$

причем выполняются следующие соотношения:

$$
\begin{aligned}
& \sum_{n=1}^{N} \epsilon^{n} \frac{\partial^{2} \psi_{j}}{\partial u^{i} \partial u^{n}} \frac{\partial^{2} \psi_{k}}{\partial u^{n} \partial u^{l}}=\sum_{n=1}^{N} \epsilon^{n} \frac{\partial^{2} \psi_{k}}{\partial u^{i} \partial u^{n}} \frac{\partial^{2} \psi_{j}}{\partial u^{n} \partial u^{l}} \\
& \sum_{n=1}^{L} \varepsilon^{n} \frac{\partial^{2} \psi_{n}}{\partial u^{i} \partial u^{j}} \frac{\partial^{2} \psi_{n}}{\partial u^{k} \partial u^{l}}=\sum_{n=1}^{L} \varepsilon^{n} \frac{\partial^{2} \psi_{n}}{\partial u^{i} \partial u^{k}} \frac{\partial^{2} \psi_{n}}{\partial u^{j} \partial u^{l}} .
\end{aligned}
$$

Работа вьполнена при финансовой поддержке Фонда Александра фон Гумболшдта (Германия), а также РФФИ (грант № 03-01-00782), программы поддержки ведущих научных школ (грант № 2185.2003.1) и INTAS (грант № 99-1782). 
Соотношения (4) и (5) хотя и существенно различаются, но похожи, и особенно интересен случай редукции, при которой соотношения (4) и (5) просто совпадают.

СлЕДСТВИЕ. В частности, если $L=N, \epsilon^{n}=\varepsilon^{n}$ для всех $n$ (или, например, $\epsilon^{n}=$ $-\varepsilon^{n}$ для всех $\left.n\right)$ и существует функция $\Phi(u)$ такая, что $\psi_{n}=\partial \Phi / \partial u^{n}$ для всех $n$, то оба соотношения (4) и (5) совпадают с уравнениями ассоциативности двумерной топологической теории поля для потенциала $\Phi(u)$ :

$$
\sum_{n=1}^{N} \varepsilon^{n} \frac{\partial^{3} \Phi}{\partial u^{i} \partial u^{j} \partial u^{n}} \frac{\partial^{3} \Phi}{\partial u^{n} \partial u^{k} \partial u^{l}}=\sum_{n=1}^{N} \varepsilon^{n} \frac{\partial^{3} \Phi}{\partial u^{i} \partial u^{k} \partial u^{n}} \frac{\partial^{3} \Phi}{\partial u^{n} \partial u^{j} \partial u^{l}} .
$$

Таким образом, любое решение уравнений ассочиативности (6), которье, как известно, совместны, интегрируемы методом обратной задачи рассеяния и обладают богатым множеством решений (см. [3]), определяет нелокальный гамильтонов оператор гидродинамического типа с плоской метрикой:

$$
L^{i j}=\epsilon^{i} \delta^{i j} \frac{d}{d x}+\sum_{n=1}^{N} \epsilon^{n} \epsilon^{i} \epsilon^{j} \frac{\partial^{3} \Phi}{\partial u^{i} \partial u^{n} \partial u^{k}} u_{x}^{k}\left(\frac{d}{d x}\right)^{-1} \circ \frac{\partial^{3} \Phi}{\partial u^{j} \partial u^{n} \partial u^{s}} u_{x}^{s},
$$

и, более того, определяет пучок согласованных гамильтоновых операторов:

$$
L_{\lambda, \mu}^{i j}=\lambda \epsilon^{i} \delta^{i j} \frac{d}{d x}+\mu \sum_{n=1}^{N} \epsilon^{n} \epsilon^{i} \epsilon^{j} \frac{\partial^{3} \Phi}{\partial u^{i} \partial u^{n} \partial u^{k}} u_{x}^{k}\left(\frac{d}{d x}\right)^{-1} \circ \frac{\partial^{3} \Phi}{\partial u^{j} \partial u^{n} \partial u^{s}} u_{x}^{s},
$$

где $\lambda и \mu-$ произвольные константы.

Аналогичное утверждение верно для любой постоянной метрики $\eta^{i j}$ у оператора. Эта метрика всегда определяет инвариантную билинейную форму на соответствующих фробениусовых алгебрах. Таким образом, в частности, с каждым фробениусовым многообразием связаны нелокальные гамильтоновы операторы вида (7) и пучки (8). Кроме того, верно и в некотором смысле обратное утверждение, а именно, если все аффииноры $w_{n}$ нелокального гамильтонова оператора (1) определяются произвольным решением $\Phi(u)$ уравнений ассоциативности $(6)$ по формуле

$$
\left(w_{n}\right)_{j}^{i}(u)=e^{n} \eta^{i s} \xi_{j}^{r} \frac{\partial^{3} \Phi}{\partial u^{n} \partial u^{s} \partial u^{r}},
$$

где $e^{n}= \pm 1, \eta^{i s}, \xi_{j}^{r}$ - произвольные невырожденные постоянные матрицы, то метрика этого гамильтонова оператора обязательно является плоской. Структурные потоки (см. [1], [4]) нелокалшного гамилтонова оператора (7) имеют вид

$$
u_{t_{n}}^{i}=\epsilon^{i} \frac{\partial^{3} \Phi}{\partial u^{i} \partial u^{n} \partial u^{k}} u_{x}^{k}
$$

и являются интегрируемыми бигамилтоновыми системами гидродинамического типа. Условие коммутации структурных потоков (7) эквивалентно опять же уравнению ассоциативности (6).

Дальнейшие резултаты, связанные с нелокальными гамильтоновыми операторами гидродинамического типа с плоскими метриками, фробениусовыми многообразиями, уравнениями ассоциативности и соответствующими интегрируемыми иерархиями, будут опубликованы в другой статье.

\section{СПИСОК ЛИТЕРАТУРЫ}

[1] Е. В. Ферапонтов // Функц. анализ и его прил. 1991. Т. 25. № 3. С. 37-49. [2] Б. А. Дубровин, С. П. Новиков // Докл. АН СССР. 1983. Т. 270. № 4. С. 781-785. [3] В. Dubrovin // Lecture Notes in Math. 1996. V. 1620. P. 120-348; hep-th/9407018 (1994). [4] A. Ya. Maltsev, S. P. Novikov // Phys. D. 2001. V. 156. № 1-2. P. 53-80; nlin.SI/0006030 (2000).

Центр нелинейных исследований при Институте теоретической физики им. Л. Д. Ландау РАН

Принято редколлегией E-mail: mokhov@mi.ras.ru, mokhov@landau.ac.ru 